\title{
Invasive Lobular Breast Carcinoma, Tubulolobular Variant
}

National Cancer Institute

\section{Source}

National Cancer Institute. Invasive Lobular Breast Carcinoma, Tubulolobular Variant. NCI Thesaurus. Code $C 97053$.

An invasive lobular carcinoma of the breast, characterized by the presence of neoplastic cells forming small tubular structures. 